\title{
The Location and Analysis of Two Heterokaryon Incompatibility (het) Loci in Strains of Aspergillus nidulans
}

\author{
By R. B. G. DALES, ${ }^{*} \dagger$ J. MOORHOUSE AND J. H. CROFT \\ Department of Genetics, The University of Birmingham, P.O. Box 363, \\ Birmingham B15 2TT, U.K.
}

(Received 13 May 1983; revised 28 June 1983)

The heterokaryon incompatibility system in Aspergillus nidulans has been investigated by parasexual methods. The use of complementary auxotrophs with a repeated serial transfer method or with a protoplast fusion technique has enabled heterokaryons and diploid strains to be recovered from heterokaryon incompatible combinations of strains. The effects of allelic interaction at heterokaryon incompatibility (het) loci on the morphologies of the heterokaryon and diploid colonies isolated are described. Parasexual analyses conducted among strains belonging to the heterokaryon compatibility groups, $\mathrm{h}-\mathrm{cGl}$ and $\mathrm{h}-\mathrm{cB}$, and the two recombinant compatibility classes, have located the het $A$ and het $B$ genes to linkage groups $\mathrm{V}$ and VI respectively.

\section{INTRODUCTION}

Heterokaryons occur spontaneously between vegetatively compatible strains of Aspergillus nidulans grown in mixed culture under non-selective conditions. The production of these 'neutral' heterokaryons (Caten \& Jinks, 1966) can be monitored if the parental strains differ for conidiospore colour as striped, heterokaryotic conidial heads can be regularly observed within the mixtures. In this way wild isolates of the species have been classified into heterokaryon compatibility (h-c) groups (Grindle, $1963 a, b$ ), with strain pairs comprised of members from different $\mathrm{h}$-c groups unable to produce spontaneous neutral heterokaryons in unselected mixed culture. Members of any one h-c group also show similarities for other characters such as cleistothecial density, radial growth rate, penicillin titre, etc. (Croft \& Jinks, 1977). Far more variation is expressed between $h-c$ groups than occurs within individual h-c groups for these characters and it seems likely that members of any one h-c group are clonally related.

The differences in vegetative compatibility have been shown to be under the control of nuclear het genes (Jinks \& Grindle, 1963) and have no significant effect on sexual outcrossing ability (Jinks et al., 1966; Butcher, 1968) such that a sexual cross between heterokaryon incompatible wild isolates leads to the production of a range of heterokaryon compatibility classes among the progeny. The classes produced are an indication of the number of het genes segregating in the cross and also that the control of this vegetative incompatibility system is heterogenic (Esser \& Blaich, 1973). The single observable phenotype of vegetative incompatibility is controlled by more than one genetic factor, and this, combined with the high meiotic recombination frequency exhibited by this species, makes it difficult to elicit any further information from sexual outcrossing data.

The ability to use parasexual methods of analysis (Pontecorvo et al., 1953; Roper, 1966) to investigate the heterokaryon incompatibility system would be advantageous, though unfortunately the system itself precludes this approach as it prevents the first stage of the parasexual

† Present address: School of Engineering and Science, Polytechnic of Central London, 115 New Cavendish Street, London W1M 8JS, U.K.

Abbreviations: $\mathrm{Cz}$, Czapek medium, CzFS, fully-supplemented Czapek medium; FPA, $p$-fluorophenylalanine. 
cycle, heterokaryon formation. It was necessary, therefore, to investigate the possible production of balanced heterokaryons between heterokaryon incompatible strains by using strong nutritional selection involving complementary auxotrophies (Moorhouse, 1977) and techniques of protoplast fusion (Dales \& Croft, 1977; Dales, 1978). The production of balanced heterokaryons and derivative diploid strains between chosen haploid, heterokaryon incompatible combinations would make possible the analysis of effects that allelic differences at individual het loci exert within such pairings. It should also prove possible to complete the parasexual analysis of haploid progeny samples derived from these diploid strains. Parasexual data obtained in this way should make possible the linkage group location of the het genes involved.

\section{METHODS}

Strains. Strains of $A$. nidulans used were the following.

7-40 proA1, yA2; pyroA5.

7-21 sUAladE20, yA2, adE20; acrA1; phenA2; pyroA4; lysB5;sB3; nicB8; riboB2.

7-23 suAladE20, yA2, adE20; acrA1; galA1; pyroA4; facA303; sB3; nicB8; riboB2.

These strains are vegetatively compatible, being derivatives of NRRL 194 . They are considered to be members of a single h-c group and have been classified as 'Glasgow' compatible strains or h-cGl (the gene symbols and locus letters used are after Clutterbuck, 1974. Strains 7-21 and 7-23 are master strains MSD and MSF respectively: McCully \& Forbes, 1965).

$1-5$ paba-1.1;w-1.3.

1-10 paba-1.3.

These strains are mutant derivatives of wild isolate 1 in the Birmingham strain collection and have been assigned to h-cB. (Gene symbols after Clutterbuck, 1974; locus designations are arbitrary, e.g. $w-1.3$ indicates the third white-sporing mutant to be isolated from wild isolate 1 . Linkage data suggest that these alleles are located at the pabaA and $w A$ loci of NRRL 194 derivatives.)

The other strains used were the following sexual cross progeny.

$$
\begin{aligned}
\text { Cross } \mathrm{JCl}= & 1-5(\mathrm{~h}-\mathrm{cB}) \times 7-40(\mathrm{~h}-\mathrm{cG} 1) \\
& \mathrm{JC} 1-7 \text { paba-1.1 } \\
& \mathrm{JC} 1-17 \text { paba-1.1 }
\end{aligned}
$$

$$
\begin{aligned}
\text { Cross JC9 }= & 1-10(\mathrm{~h}-\mathrm{cB}) \times 7-21(\mathrm{~h}-\mathrm{cGl}) \\
& \mathrm{JC} 9-8 \text { paba-1.3; acr } \mathrm{AI}
\end{aligned}
$$

These crosses liberated four compatibility classes in equal frequency indicating that two unlinked het genes, het $A$ and het $B$, were segregating among the progeny (Croft \& Jinks, 1977). The four compatibility classes have been given the following arbitrary het allele designations: [het $A I$; hetBI], compatible with the h-cGl parent; [het $A 2$; het $B 2]$, compatible with the h-cB parent; [het $A 1$; het B2] and [het $A 2$; het $B 1]$, the two recombinant classes.

$\mathrm{H}-\mathrm{c}$ groupings have been extended to include inter-h-c group progeny strains which are heterokaryon compatible with their respective h-c group isolates.

Media. Czapek medium $(\mathrm{Cz})$ was routinely used as a minimal medium. Auxotrophic strains were cultured on $\mathrm{Cz}$ supplemented with the particular nutrient(s) required.

Strain culture. General culture methods and methods for the production and analysis of sexual crosses were derived from those of Pontecorvo et al. (1953) and Clutterbuck (1974).

Compatibility testing. Heterokaryon compatibility tests were routinely performed on fully-supplemented $\mathrm{Cz}$ medium (CzFS). Pairs of strains bearing a conidial colour difference were point inoculated together and incubated at $35^{\circ} \mathrm{C}$ for $3 \mathrm{~d}$. The colonies were then scored ( $\times 100$ magnification) for the presence or absence of striped, heterokaryotic conidial heads.

Standard method for the production of balanced heterokaryons. Heterokaryons do not persist in $A$. nidulans unless they are maintained by selection pressure. To produce them, strains bearing complementary auxotrophic mutations were used. Conidial colour mutants were used to visualize parental contributions to the heterokaryons. Spore suspensions $\left(10^{6}-10^{7}\right.$ spores $\left.\mathrm{ml}^{-1}\right)$ of the parental strains were prepared and $0 \cdot 1 \mathrm{ml}$ aliquots spread together on $\mathrm{Cz}$ plates. These were incubated at $35^{\circ} \mathrm{C}$ until growth was observed. Mass hyphal transfers in agar blocks were made to fresh $\mathrm{Cz}$ plates from centres of growth on which both parental spore colours were visible. These plates were incubated at $35^{\circ} \mathrm{C}$ for up to 1 week and scored for balanced heterokaryon production. In experiments involving serial subculture a maximum of four further mass hyphal transfers were employed, with each transfer being incubated for 1 week at $35^{\circ} \mathrm{C}$ and then the next subculture made from areas showing slight growth and mixed parental spore colours. 
Protoplast methods. Methods for the production, fusion and regeneration of protoplasts have been described previously (Dales \& Croft, 1977).

Isolation of diploid strains. Diploid isolation was achieved by plating dense conidial suspensions, or mass hyphal suspensions from colonies with sparse conidiation, obtained from heterokaryons in soft $\mathrm{Cz}$ overlayers on $\mathrm{Cz}$ agar plates and incubating at $35^{\circ} \mathrm{C}$.

Haploidization of diploid strains. Diploid colonies were induced to haploidize from small colony centres on CzFS agar containing either $p$-fluorophenylalanine (FPA) (Morpurgo, 1961; Lhoas, 1961) or benomyl ('Benlate', E. 1. du Pont de Nemours \& Co., U.S.A.; marketed by Pan Britannica Industries Ltd., Waltham Cross, Herts., U.K.) (Hastie, 1970; Edwards et al., 1975). It should be noted that, for parasexual analysis, only one segregant sector per haploidizing colony centre was collected to avoid the possibility of resampling the same, or the reciprocal product of the same event.

Throughout this paper the terms compatible and incompatible when used will mean heterokaryon compatible and heterokaryon incompatible respectively.

\section{RESULTS}

Initial experiments which attempted to produce balanced heterokaryons by a standard method between h-c groups B and Gl involved pairs of strains possessing selectable auxotrophic markers and visible conidial colour differences. Balanced heterokaryons could be isolated from within but not from between $h-c$ group pairings. These results confirm the previous observations of non-selected (neutral) heterokaryons by which the h-c groupings were originally classified (Grindle, 1963a,b).

Further attempts were made to produce balanced heterokaryons between strains of different heterokaryon compatibility by serial subculture from areas of mixed inocula on $\mathrm{Cz}$ agar. Out of twelve combinations where there was an allelic difference at het $A$ only, heterokaryons were recovered from five. These were similar to heterokaryons produced from compatible strain combinations with regions of rapid growth and heterokaryotic conidial heads visible. Conidial suspensions obtained from these heterokaryons plated in soft $\mathrm{Cz}$ agar overlayers led to the recovery of diploid colonies. Some spontaneous diploids were also isolated from the serial subculture plates. These diploids resembled compatible strain combination diploids in colony morphology, mean spore diameter and conidial coloration; however, a reduction in sporehead density was observed. One such diploid, isolated from the parental strains 7-23 and JCl-7, was haploidized on CzFS + FPA and a haploid progeny sample of 50 yellow-sporing segregants collected and analysed. The results are presented in Table 1. It can be seen that het $A$ shows complete mitotic linkage with facA on linkage group $\mathrm{V}$.

In pairings which differed at the hetB locus typical balanced heterokaryons were not produced. After repeated subculture faster-growing sectors were sometimes recovered and these were of three basic types. Type 1 were composed of a loose mycelial network with much of the hyphal extension being below the agar surface and having extremely poor conidiation. The

Table 1. Segregation of hetA among haploid segregants from the diploid 7-23/JC1-7

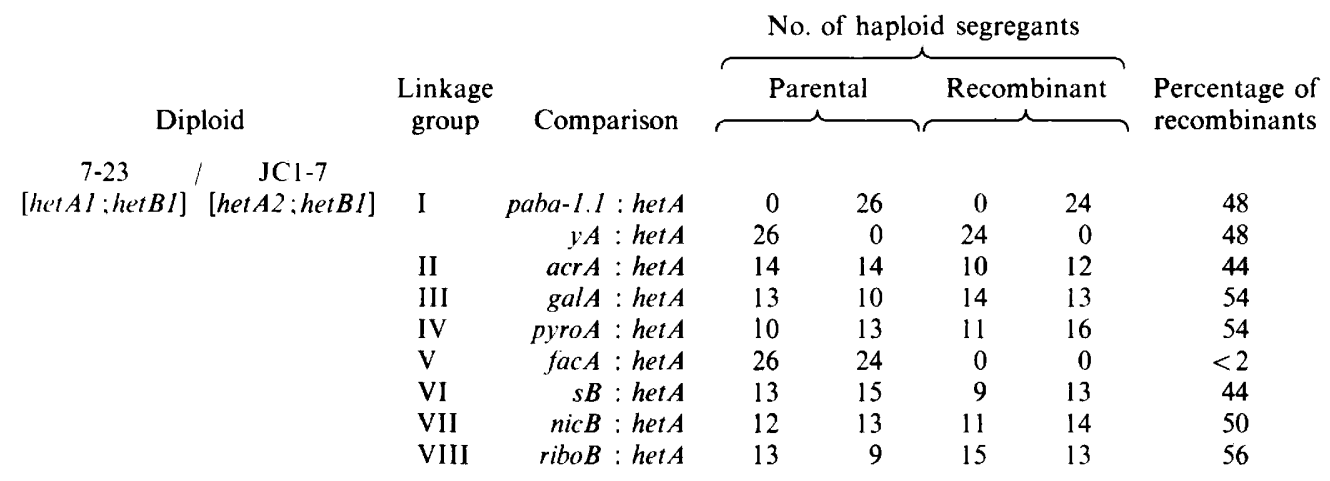


Table 2. Segregation of het genes among haploid segregants from the diploids 7-21/JC1-17 and 7-21/JC9-8

All other joint segregations did not depart from expected values. Moreover the allelic ratios obtained were not significantly different from $1: 1$ and this random assortment indicates a translocation-free relationship (Pontecorvo \& Käfer, 1958; Käfer, 1958) between the parental strains.

\begin{tabular}{|c|c|c|c|c|c|c|c|}
\hline \multirow[b]{2}{*}{ Diploid } & & & \multicolumn{4}{|c|}{ No. of haploid segregants } & \multirow[b]{2}{*}{$\begin{array}{l}\text { Percentage of } \\
\text { recombinants }\end{array}$} \\
\hline & $\begin{array}{l}\text { Linkage } \\
\text { group }\end{array}$ & Comparison & \multicolumn{2}{|c|}{ Parental } & \multicolumn{2}{|c|}{ Recombinant } & \\
\hline $\begin{array}{cc}7-21 & \mathrm{JC} 1-17 \\
{[\text { het } A 1 ; \text { het } B 1]} & {[\text { het } A 1 ; \text { het } B 2]}\end{array}$ & VI & $s B:$ het $B$ & 22 & 18 & 0 & 0 & $<2$ \\
\hline $\begin{array}{c}7-21 / \text { JC9-8 } \\
{[\text { het } A 1 ; \text { het } B I]}\end{array}$ & $\begin{array}{l}\text { V } \\
\text { VI }\end{array}$ & $\begin{aligned} \text { lys } B & : \text { het } A \\
s B & : \text { het } B\end{aligned}$ & $\begin{array}{l}51 \\
49\end{array}$ & $\begin{array}{l}45 \\
47\end{array}$ & $\begin{array}{l}1 \\
0\end{array}$ & $\begin{array}{l}0 \\
1\end{array}$ & $\begin{array}{l}1 \\
1\end{array}$ \\
\hline
\end{tabular}

sectors and the surrounding agar were suffused with a deep pinkish-red pigmentation. Type 1 colonies readily sectored out areas of both parental homokaryons when subcultured on to CzFS agar. Type 2 sectors were mycelial mats with a deep pink, cell-localized pigmentation and sparse conidiation. The sporeheads produced were small and of a light green coloration with the diffuse morphology typical of diploid strains. The mean conidiospore diameter was significantly larger than that of haploid conidia. Subcultures displayed a uniform radial growth and did not segregate parental homokaryons on CzFS agar. Finally type 3, which also appeared as sectors emanating from type 1 and type 2 colonies, were indistinguishable from typical diploid strains. Soft agar platings for diploid isolation of a mixed conidial and hyphal suspension obtained from type 1 colonies led to the recovery of both type 2 and type 3 colonies. These observations indicate that type 1 colonies are heterokaryotic and that types 2 and 3 arise within type 1 as a result of nuclear fusion events.

A single type 3 normal diploid morphology sector was recovered from a pairing of strains 7-23 and JC1-17. This was purified, haploidized on CzFS + FPA and a sample of 61 haploid segregants collected and their genotypes determined. There was no segregation of the alleles at the $s B$ locus, with all the progeny bearing the JC1-17-derived wild-type allele. Furthermore all the progeny possessed the JC1-17-derived allele at the compatibility locus het $B$, being of the compatibility type [hetA1; hetB2]. All the other marker loci displayed typical segregation patterns. It was provisionally concluded that $s B$ and het $B$ were located on the same linkage group (linkage group VI). Presumably, homozygosity had been achieved for part, or all, of this linkage group either by mitotic recombination or by whole-chromosome non-disjunction (Käfer, 1961 ) which had removed the deleterious effects of heterozygosity at the het $B$ locus, resulting in the outgrowth of a normal morphology diploid sector.

Inoculation of type 2 colonies on to CzFS + FPA also yielded haploid segregants with normal morphology. This facilitated the collection of a progeny sample of 40 haploid strains from the initial pairing of strains 7-21 and JC1-17 (Table 2). Segregations were obtained for both $s B$ and het $B$, with these loci showing complete mitotic linkage confirming the location of het $B$ on linkage group VI. Reassortment was obtained for all linkage groups among the progeny sample which would indicate that the type 2 colonies are diploid.

Serial subculture from a mixture of strains of Gl and B compatibilities enabled the recovery of a single faster-growing sector and this closely resembled the type 2 colonies described above, with sectors of normal diploid morphology also being produced. Haploidizational analysis of these normal diploid morphology sectors revealed a lack of segregation for both the het $A$ and het $B$ loci.

It has proved possible to isolate heterokaryons and diploids from $\mathrm{h}-\mathrm{cB} / \mathrm{h}-\mathrm{cGl}$ pairings by protoplast fusion methods (Dales \& Croft, 1977). The morphologies of the heterokaryons and diploids recovered were very similar, respectively, to the type 1 and type 2 colonies already described. A type 2-like colony from a combination between strains 7-21 and JC9-8 was induced 
to haploidize on CzFS medium containing benomyl. Analysis of the segregants obtained (Table 2) directly confirmed the location of hetA and hetB on linkage groups V and VI respectively.

Further protoplast fusion experiments have been performed (data not shown) where different combinations of markers and overall background genotype were employed. Also, in situations where unlike alleles were operative at only one het locus then both different 'homozygous' combinations of alleles at the other het locus have been used. In all cases the morphological observations for the heterokaryons and diploid colonies recovered have been in agreement with those reported here. Investigation of diploids which are heterozygous for compatibility at het $A$ only has confirmed that this leads to a noticeable reduction in sporehead density, as it has proved possible to identify sectors of typical diploid morphology emanating from these colonies. Analysis of haploid segregants indicated that these normal diploid sectors were homozygous at the hetA locus.

\section{DISCUSSION}

Allelic differences at both het $A$ and het $B$ are effective in the prevention of neutral heterokaryosis and therefore a single het gene difference can be enough to prevent heterokaryon formation. This is not to say that hyphal anastomoses and neutral heterokaryons do not occur under non-selective conditions but that they were not observed to manifest their presence in the production of striped conidial heads. The successful recovery of heterokaryons and diploids from vegetatively incompatible parents by prolonged nutritional selection would indicate that hyphal anastomoses do take place and one possibility is that the anastomosis frequency is reduced in incompatible pairings. Balanced heterokaryons and diploids were readily recovered from strain pairs differing at het $A$ but were more difficult to isolate from pairs differing at het $B$, suggesting that the allelic difference at $h e t B$ is more effective in depressing the frequency of hyphal anastomosis. For the parental strain combination of h-cB with h-cGl, heterokaryons were not recovered and only a single diploid colony was isolated suggesting that these het genes may act with a degree of additivity in the prevention of heterokaryosis.

It is apparent that the forced pairing of strains of different heterokaryon compatibility can have profound morphological effects upon the association. Varying the genetic background, while retaining the same combinations of het genes, has no observable effect. Consequently the abnormal morphologies must be attributable to the expression of interacting alleles at het loci following either hyphal or protoplast fusion. Allelic interaction at het $B$ imparts a far greater degree of morphological abnormality than does the interaction of unlike alleles at het $A$ and this would imply that het genes do not all have the same mode of action or operate in the same area of cellular metabolism.

The presence of apparently normal diploid sectors within diploids heterozygous at het loci is thought to indicate selection pressure favouring genetical events leading to homozygosity at het genes. This implies that differences at these loci impart a considerable physiological constraint upon the association even if, as is the case with het $A$, the observable effects on heterokaryon and diploid morphologies are not extreme. This constraint may lead to selection against the products of hyphal anastomosis formed between incompatible strains in mixed cultures under both nutritionally selective and non-selective conditions, and would account for the observed results without a need for a decrease in the frequency of anastomosis.

Protoplast fusion was highly successful in the regular production of heterokaryons from parents differing at either, or both, the het $A$ or het $B$ genes. This technique effectively provides a source of artificially induced anastomoses which, at the appropriate dilution, become discrete colony-forming units capable of regeneration and outgrowth in the absence of competition from parental mycelia.

Finally, there can be little doubt that het $A$ and het $B$ are located on linkage groups $\mathrm{V}$ and VI respectively. Preliminary meiotic mapping data suggest that het $A$ is loosely linked to the riboD locus.

We acknowledge the Science Research Council for the award of research studentships to J.M. \& R.B.G.D. 


\section{REFERENCES}

BUTCHER, A. C. (1968). The relationship between sexual outcrossing and heterokaryon incompatibility in Aspergillus nidulans. Heredity 23, 443-452.

CATEN, C. E. \& Jinks, J. L. (1966). Heterokaryosis: its significance in wild homothallic Ascomycetes and Fungi Imperfecti. Transactions of the British Mycological Society 49, 81-93.

Clutterbuck, A. J. (1974). Aspergillus nidulans. In Handbook of Genetics, vol. 1, pp. 447-510. Edited by R. C. King. New York: Plenum Press.

Croft, J. H. \& JinKs, J. L. (1977). Aspects of the population genetics of Aspergillus nidulans. In Genetics and Physiology of Aspergillus, pp. 339-360. Edited by J. E. Smith \& J. A. Pateman. London: Academic Press.

DALES, R. B. G. (1978). Heterokaryon incompatibility in Aspergillus nidulans. Ph.D. thesis, Birmingham University, U.K,

Dales, R. B. G. \& CROFT, J. H. (1977). Protoplast fusion and the isolation of heterokaryons and diploids from vegetatively incompatible strains of Aspergillus nidulans. FEMS Microbiology Letters 1, 201-204.

Edwards, G. F. St L., Normansell, I. D. \& Holt, G. (1975). Benlate induced haploidisation in diploid strains of Aspergillus nidulans and Penicillium chrysogenum. Aspergillus Newsletter 12, 15.

ESSER, K. \& Blaich, R. (1973). Heterogenic incompatibility in plants and animals. Advances in Genetics 17, 107-152.

GRINDLE, M. (1963a). Heterokaryon compatibility of unrelated strains in the Aspergillus nidulans group. Heredity 18, 191-204.

GRINDLE, M. (1963b). Heterokaryon compatibility of closely related wild isolates of Aspergillus nidulans. Heredity 18, 397-405.

HASTIE, A. C. (1970). Benlate-induced instability of Aspergillus diploids. Nature, London 226, 771.
JinKs, J. L. \& GrindLE, M. (1963). The genetical basis of heterokaryon incompatibility in Aspergillus nidulans. Heredity 18, 407-411.

Jinks, J. L., Caten, C. E., Simchen, G. \& Croft, J. H. (1966). Heterokaryon incompatibility and variation in wild populations of Aspergillus nidulans. Heredity 21, 227-239.

KÄFER, E. (1958). An 8-chromosome map of Aspergillus nidulans. Advances in Genetics 9, 105-145.

KÄFER, E. (1961). The process of spontaneous recombination in vegetative nuclei of Aspergillus nidulans. Genetics 46, 1581-1609.

LHOAS, P. (1961). Mitotic haploidization by treatment of Aspergillus niger diploids with para-fluorophenylalanine. Nature, London 190, 744.

MCCully, K. S. \& ForbeS, E. (1965). The use of $p$ fluorophenylalanine with 'master strains' of Aspergillus nidulans for assigning genes to linkage groups. Genetical Research 6, 352-359.

MOORHOUSE, J. (1977). The genetical basis of heterokaryon incompatibility in Aspergillus nidulans. Ph.D. thesis, Birmingham University, U.K.

MORPURGO, G. (1961). Somatic segregation induced by p-fluorophenylalanine. Aspergillus Newsletter 2, 10.

PONTECORVo, G. \& KÄFER, E, (1958). Genetic analysis based on mitotic recombination. Advances in Genetics 9, 71-104.

Pontecorvo, G., Roper, J. A., Hemmons, L. M., Macdonald, K. D. \& Bufton, A. W. J. (1953). The genetics of Aspergillus nidulans. Advances in Genetics 5, 141-238.

ROPER, J. A. (1966). Mechanisms of inheritance: the parasexual cycle. In The Fungi, vol. 2, pp. 589-617. Edited by G. C. Ainsworth \& A. S. Sussman. New York: Academic Press. 